\title{
Letter
}

\section{Efficient Photon Recycling and Radiation Trapping in Cesium Lead Halide Perovskite Waveguides}

Ibrahim Dursun, Yangzi Zheng, Tianle Guo, Michele De Bastiani, Bekir Turedi, Lutfan Sinatra, Md Azimul Haque, Bin Sun, Ayan A. Zhumekenov, Makhsud I. Saidaminov, F. Pelayo Garcia de Arquer, Edward H. Sargent, Tom Wu, Yuri N Gartstein, Osman M. Bakr, Omar F. Mohammed, and Anton V. Malko

ACS Energy Lett., Just Accepted Manuscript • DOI: 10.1021/acsenergylett.8b00758 • Publication Date (Web): 25 May 2018

Downloaded from http://pubs.acs.org on May 29, 2018

\section{Just Accepted}

"Just Accepted" manuscripts have been peer-reviewed and accepted for publication. They are posted online prior to technical editing, formatting for publication and author proofing. The American Chemical Society provides "Just Accepted" as a service to the research community to expedite the dissemination of scientific material as soon as possible after acceptance. "Just Accepted" manuscripts appear in full in PDF format accompanied by an HTML abstract. "Just Accepted" manuscripts have been fully peer reviewed, but should not be considered the official version of record. They are citable by the Digital Object Identifier (DOI@). "Just Accepted" is an optional service offered to authors. Therefore, the "Just Accepted" Web site may not include all articles that will be published in the journal. After a manuscript is technically edited and formatted, it will be removed from the "Just Accepted" Web site and published as an ASAP article. Note that technical editing may introduce minor changes to the manuscript text and/or graphics which could affect content, and all legal disclaimers and ethical guidelines that apply to the journal pertain. ACS cannot be held responsible for errors or consequences arising from the use of information contained in these "Just Accepted" manuscripts. 


\title{
Efficient Photon Recycling and Radiation Trapping in Cesium Lead Halide Perovskite Waveguides
}

Ibrahim Dursun ${ }^{1,2}$, Yangzi Zheng ${ }^{3}$, Tianle Guo ${ }^{3}$, Michele De Bastiani ${ }^{1}$, Bekir Turedi ${ }^{1,2}$, Lutfan Sinatra $^{1,2}$, Md Azimul Haque ${ }^{4}$, Bin Sun ${ }^{5}$, Ayan A Zhumekenov ${ }^{1,2}$, Makhsud I. Saidaminov ${ }^{5}$, F. Pelayo García de Arquer ${ }^{5}$, Edward H. Sargent ${ }^{5}$, Tom Wu ${ }^{4}$, Yuri N. Gartstein ${ }^{3}$, Osman M. Bakr ${ }^{1,2}$, Omar F. Mohammed ${ }^{1 *}$, Anton V. Malko ${ }^{3 *}$

${ }^{1}$ KAUST Solar Center, Division of Physical Sciences and Engineering, King Abdullah University of Science and Technology, Thuwal 23955-6900, Saudi Arabia

${ }^{2}$ KAUST Catalysis Center, Division of Physical Sciences and Engineering, King Abdullah University of Science and Technology, Thuwal 23955-6900, Saudi Arabia

${ }^{3}$ Department of Physics, the University of Texas at Dallas, Richardson, TX, 75080, USA

${ }^{4}$ Materials Science and Engineering, Division of Physical Sciences and Engineering, King Abdullah University of Science and Technology, Thuwal 23955-6900, Saudi Arabia

${ }^{5}$ Department of Electrical and Computer Engineering, University of Toronto, Toronto, Ontario M5S 3G4, Canada

*Email: anton.malko@utdallas.edu and omar.abdelsaboor@kaust.edu.sa

\begin{abstract}
Cesium lead halide perovskite materials have attracted considerable attention for potential applications in lasers, light emitting diodes and photodetectors. Here, we provide the experimental and theoretical evidence for photon recycling in $\mathrm{CsPbBr}_{3}$ perovskite microwires. Using twophoton excitation, we recorded photoluminescence (PL) lifetimes and emission spectra as a function of the lateral distance between PL excitation and collection positions along the microwire,
\end{abstract}


with separations exceeding $100 \mu \mathrm{m}$. At longer separations, the PL spectrum develops a red-shifted emission peak accompanied by an appearance of well-resolved rise times in the PL kinetics. We developed quantitative modeling that accounts for bimolecular recombination and photon recycling within the microwire waveguide and is sufficient to account for the observed decay modifications. It relies on a high radiative efficiency in $\mathrm{CsPbBr}_{3}$ perovskite microwires and provides crucial information about the potential impact of photon recycling and waveguide trapping on optoelectronic properties of cesium lead halide perovskite materials.

\section{Graphical table of content (TOC)}

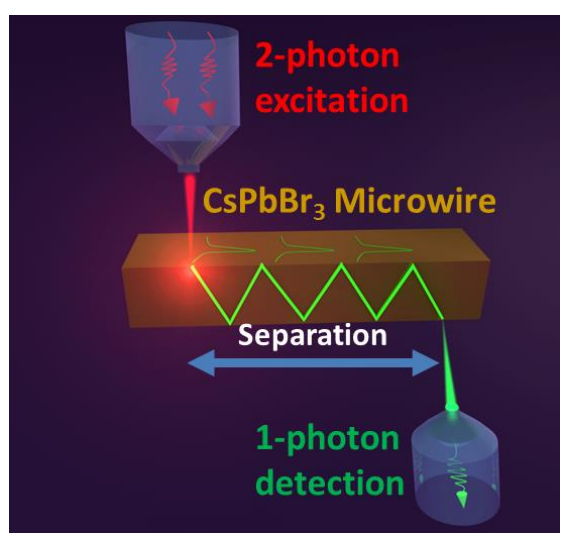


Solution-processed organic-inorganic hybrid perovskite-based semiconductors have rapidly progressed as versatile and promising materials for low-cost and high-performance optoelectronics applications in the past five years ${ }^{1-11}$ due to their remarkable light absorption ${ }^{12}$, long carrier diffusion length ${ }^{12}$, 13 , controllable dimensionalities ${ }^{14-21}$ and tunable bandgaps. ${ }^{22}$ Recently, all inorganic $\mathrm{CsPbX}_{3}\left(\mathrm{X}=\mathrm{Br}^{-}, \mathrm{I}^{-}\right.$or $\left.\mathrm{Cl}^{-}\right)$perovskites have attracted particular attention for light emitting diodes (LEDs) ${ }^{23,24}$ and lasers ${ }^{25-27}$ due to enhanced light emission and photo/thermal stability. ${ }^{28,} 29$ The wavelength tunability of photoluminescence (PL) emission can be further manipulated via the choice of the desired halide precursor or anion exchange reactions. Facile, solution-processable methods allow these materials to be fabricated in a variety of configurations such as nanowires, ${ }^{30-32}$ nanocrystals, ${ }^{33,} 34$ and microrods. ${ }^{35}$ Wavelength-scale sizes of these microstructures make them suitable for potential integration with on-chip, miniaturized devices such as coherent light sources, LEDs and photodetectors.

Laser and LED applications of $\mathrm{CsPbX}_{3}$ materials require detailed knowledge of the dynamics of mode propagation and spectral redistribution of the emission in the waveguiding structures. Repeated incoherent absorption and re-emission of photons in strongly absorbing media is expected to substantially affect carrier recombination dynamics and mode propagation, influencing quantum efficiency and light dynamics. Significant processes of self-absorption and light trapping have been observed in solid state materials with high PL quantum yields (PLQY) such as III-V semiconductors $(\mathrm{GaAs})^{36-38}$ and rare-earth doped fibers, ${ }^{39,40}$ leading to large modifications of the emission lineshape and significantly affecting emission lifetime measurements. Photon recycling, i.e. numerous iterative self-absorbance and photon re-emissions events within the photoactive layer has been known to substantially enhance the photovoltaic conversion efficiency of GaAs 
solar cells and photodetectors, ${ }^{41,42}$ although its importance for high efficiency perovskite-based solar cells has been the subject of recent controversy. ${ }^{43-45}$

In this work, we performed a detailed time- and spectrally-resolved investigation of waveguideassisted photon self-absorption/re-emission in single crystal $\mathrm{CsPbr}_{3}$ microwires synthesized via one-step, solution-processing method. We took advantages of two-photon excitation to avoid surface related PL quenching and moisture induced artefacts. ${ }^{46,47}$ We directly tracked the evolution of PL signals as a function of the lateral separation between excitation and collection spots along the microwire to the distances exceeding $100 \mu \mathrm{m}$. We observed appearance of the delayed rises in PL kinetics, accompanied by continuous spectral re-distribution of the PL signal, initially centered at $525 \mathrm{~nm}$, towards lower energy, concentrating the emission intensity in a well-defined peak at $545 \mathrm{~nm}$. To explain salient features of the position-dependent PL dynamics, we numerically analyzed a model of the evolution of charge carrier density that accounts for radiation trapping in 1D waveguides. The model establishes a quantitative framework that accommodates well the observed PL kinetics and unambiguously points to the importance of photon recycling effects. Moreover, it indicates that internal radiative efficiency of the $\mathrm{Cs} \mathrm{PbBr}_{3}$ microwires is close to unity, thus providing the necessary foundation for the usage of $\mathrm{CsPbBr} 3$ perovskite materials in lightemitting applications. 
Results and Discussions: Sample preparation and structural characterization of $\mathrm{Cs}_{\mathrm{PbBr}}$ microwires. We synthesized $\mathrm{CsPbBr}_{3}$ microwires using a customized cast-capping crystallization ${ }^{48}$ process. Several drops of $\mathrm{CsPbBr}_{3}$ precursor solution were dropped on a glass substrate, while a second glass was used as the capping substrate to confine the solution. The two glasses were heated at $100^{\circ} \mathrm{C}$ for 3 to 5 days to let the crystals grow up to the desired sizes. The resulting arrays of microwires were characterized using optical spectroscopy, scanning electron microscopy (SEM), $\mathrm{X}$-ray diffraction (XRD), transmission electron microscopy (TEM), selected area electron diffraction (SAED) and Energy dispersive X-ray spectroscopy (EDX) techniques (Figure 1 and
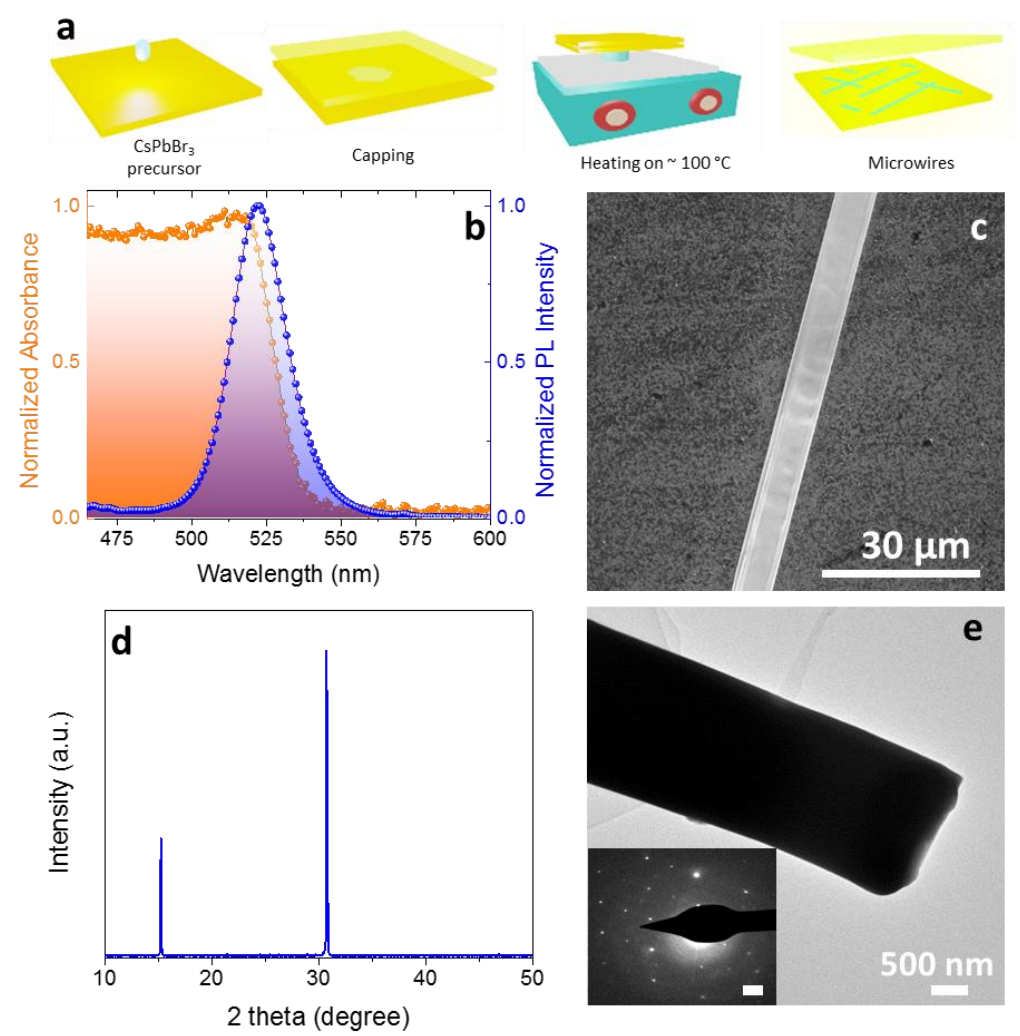

Figure 1. Synthesis and characterization of $\mathrm{CsPbBr}_{3}$ microwires. (a) Schematic drawing of cast-capping method for growing the $\mathrm{CsPbBr}_{3}$ microwires. (b) Normalized absorption and photoluminescence spectra of $\mathrm{CsPbBr}_{3}$ microwire crystals grown by cast capping method. (c) $\mathrm{SEM}$ top view of a single $\mathrm{CsPbBr}_{3} \mathrm{Picrowire}$ with a scale bar of $30 \mu \mathrm{m}$. (d) XRD spectra of as grown $\mathrm{CsPbBr}_{3}$ microwires. (e) TEM image of single microwires with a scale bar of $500 \mathrm{~nm}$. The inset shows the high-resolution SAED results of a single $\mathrm{CsPbBr}_{3}$ microwire with a scale bar of $2 \mathrm{~nm}^{-1}$. 
Figure S1) that demonstrate nearly ideal crystal structure, stoichiometric composition and shape uniformity, making microwires ideally suited for light trapping and waveguiding.

Bulk optical absorption and PL emission measurements show the onset of the optical bandgap at $\sim 510 \mathrm{~nm}$ and main PL peak at $\sim 525 \mathrm{~nm}$, with a full-width half-maximum (FWHM) of $21 \mathrm{~nm}$ when the $\mathrm{CsPbBr}_{3}$ microwires are excited at $400 \mathrm{~nm}$, Figure 1(b). The structural analysis confirms the crystalline nature of the microwires. Figure 1(c) shows the top-view SEM image of assynthesized microwires. The microwires present several different lengths, from few to hundreds of micrometers, according to the synthesis protocol, with a uniform width and thickness of 5-7 $\mu \mathrm{m}$. The XRD spectrum of the as-synthesized microwires shows two sharp peaks at $15^{\circ}$ and $30^{\circ}$, in good agreement with the orthorhombic $\mathrm{CsPbBr}_{3}$ phase, indicating the preferential orientation of the crystallization process. Furthermore, TEM and selected area electron diffraction (SAED) patterns confirm the crystalline nature of the wires and the orthorhombic symmetry, Figure 1(e). Finally, EDX analysis probed the elemental uniformity of as-synthesized microwires with a homogeneous distribution of $\mathrm{Cs} / \mathrm{Pb} / \mathrm{Br}(\sim 1 / 1 / 3)$ matching well the stoichiometry of $\mathrm{CsPbBr}_{3}$ (Figure S1). All these results collectively confirmed that high quality single crystalline $\mathrm{CsPbBr}_{3}$ microwires with preferential orientation were obtained through our customized cast-capping technique.

Photon waveguiding and absorption/re-emission. The primary parameters that govern photon recycling are extinction coefficient $(\alpha)$ and radiative efficiency within individual microwires. ${ }^{49}$ When absorption length $(l=1 / \alpha)$ is much smaller than the size of the sample, the light's propagation between the excitation and detection regions will allow for many absorption/re-emission events, thus influencing the spectrum shape and modifying the observed lifetime. Self-absorption is assisted by total internal reflection (TIR) that keeps a substantial fraction of photons from escaping 
the structure and guides light over longer distances in the waveguide. Bulk absorption measurements are not representative of the absorption within individual microwires due to the non-continuous microwires' coverage on the glass substrate. To alleviate this problem, we performed linear absorption measurements on individual microwires using confocal double objective geometry as shown in the left part of the inset in Figure 2(a). An objective with a lower numerical aperture (NA 0.5) has been used to couple broadband white light (WL) through the top facet of the microwire (from the air side), while higher-NA objective positioned below the quartz substrate was used for light collection. The thickness of the microwires (as seen in SEM imaging) has been consistently found in the $\sim 5$ um range, thus allowing us to estimate the absorption coefficient. The use of low-NA objective largely avoids the problem of WL coupling into the waveguiding modes and thus escaping detection ( $\mathrm{NA}=0.5$ corresponds to $30^{\circ}$ coupling half-angle, while the index of refraction $n \sim 2.3$ of the $\mathrm{CsPbBr}_{3}$ material corresponds to TIR critical angle of $\theta_{c r} \sim 27^{\circ}$ ). Figure 2(a) indicates that optical density (OD) of individual microwires ranges from 0.1-0.15 OD at the main exciton peak and approximately between 0.05-0.075 at the PL emission wavelengths of $525-530 \mathrm{~nm}$. The corresponding absorption length $l$ is on the order of $20-40 \mu \mathrm{m}$ and extinction coefficient $\alpha$ in the range $\sim(2.5-5) * 10^{2} \mathrm{~cm}^{-1}$. The extinction values obtained in our work point to the potential contribution of the TIR-assisted waveguiding to photon recycling in microwires. 

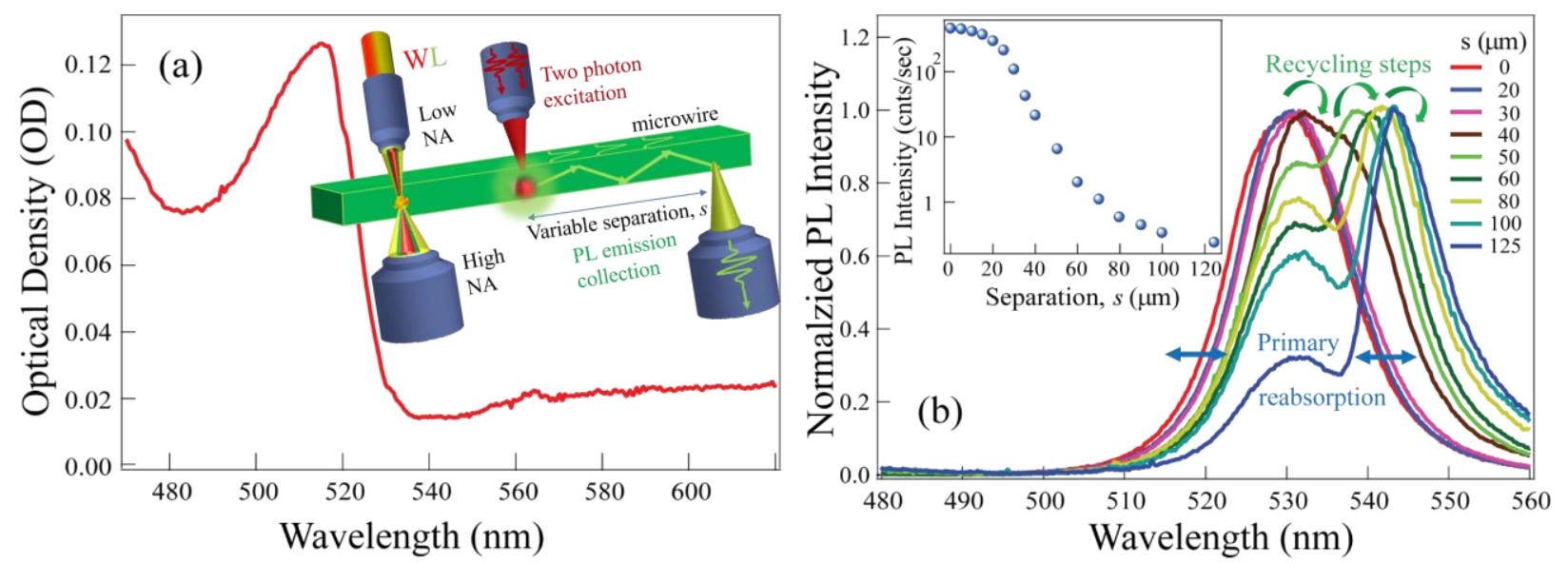

Figure 2: (a) Linear absorption spectra of an individual microwire. Inset: Left part - schematics of linear absorption measurements using white light. Right part - variable length excitation/collection measurements using the same pair of objectives, with the bottom one translated along the microwire. (b) PL spectra as function of separation $s$. Spectra are normalized at the maximum emission intensity. Excitation power $20 \mu \mathrm{W}$. Inset: PL emission intensity at the 530 $\mathrm{nm}$ peak as a function of separation distance $s$.

PL spectra indicating photon recycling. Figure 2(b) presents PL spectra for several values of the spatial separation $s$ between the excitation and collection volumes along the individual microwire. Same excitation/collection arrangement (right side of the inset in Figure 2(a)) has been used, whereas excitation light is $800 \mathrm{~nm}$ laser positioned in the middle of the microwire and PL collection spot is scanned along the microwire's bottom surface, with separation distance $s$ in excess of $100 \mu \mathrm{m}$. The relative weight of the main emission peak at $\sim 530 \mathrm{~nm}$ at first shifts toward longer emission wavelength (for $s=0-30 \mu \mathrm{m}$ separations, indicated by arrows). This initial shift is attributed to the primary (i.e., PL emission that is produced by direct laser excitation) photon reabsorption at shorter wavelengths and correspondingly larger PL contribution at longer wavelength due to spectral filtering within the perovskite material as previously described. ${ }^{50}$ However, at longer separation distances we observe the appearance and relative growth of a welldefined peak at the lower energy, that is continuously evolving to $\sim 545 \mathrm{~nm}$ as separation distance exceeds $100 \mu \mathrm{m}$. It is clearly seen that while the overall intensity of the emission is dropping off 
sharply (inset to Figure 2(b)), the re-emission peak is taking over the primary PL emission. Similar behavior of PL spectra has been recently observed in Ref. 32. This behavior is indicative of repetitive PL self-absorption/re-emission mechanism under conditions of inhomogeneous broadening that is shifting emission down in energy (following sharp cutoff in absorption profile) and points towards multistep photon recycling in $\mathrm{CsPbr}_{3}$ perovskites.

Quantitative description of photon recycling using PL dynamics. Quantitative interpretation of the PL emission spectra is complicated by the specifics of light propagation within the microwire. Visual appearance and intensity of PL peaks are often related to the presence of macroscopic surface defects that strongly influence the amount of the collected PL light, Figure S2. At the same time, PL lifetime measurements are only affected by the intrinsic factors such as pumping of the perovskite by the two-photon excitation pulse at short separation distances and pumping via PL absorption/re-emission events at longer separations. Using $800 \mathrm{~nm}$ laser for two-photon excitation allows us to mostly avoid excitation of the surface-related states that typically manifest themselves in very short initial PL lifetime components (some examples are shown in Supporting Information). ${ }^{49}$ Figure 3(a) shows that, as the separation is increased, we observe an appearance of a delayed rise components, indicating the influx of excitations to the distant regions of the microwire. To properly analyze and represent the dynamics in a noise-free format, we consistently fitted PL decays with 2-3 exponentials, fully accounting for the observed rise and fall dynamics. Figure 3(b) shows an expanded view of the fitted kinetics at different separation distances for shorter delay times. The data illustrate the gradual evolution of the rise time components in the PL dynamics at longer separations. This behavior is a clear signature of the delayed energy influx into the remote regions of the microwire. 
In general, there could be two mechanisms generating excitations in the remote parts of the microwire away from the original laser excitation spot. One is the photon recycling, and the other is carrier diffusion, where charge carriers generated by the laser pulse diffuse within the wire and recombine at the later time, giving rise to the delayed PL emission. ${ }^{51}$ To clarify this issue, we performed independent carrier mobility measurements in individual microwires using two dimensional space charge limited current (2D-SCLC) and field effect transistor (FET) methods (more details are in Experimental Section and Supporting Information, Figure S3 and S4). The results indicate that the hole mobilities are in the range of $\mu=0.01-0.02 \mathrm{~cm}^{2} /(\mathrm{Vs})$ which is in a good agreement with the value reported by Huo et al. ${ }^{52}$ Considering PL lifetime of the carriers to be around $400 \mathrm{~ns}$ (Figure 3(a)), the estimated room-temperature diffusion length, $L_{D} \sim 0.1 \mu \mathrm{m}$. Hence, the observed evolution of PL decay dynamics at separation distances of tens of $\mu \mathrm{m}$ cannot be a result of charge carrier diffusion. The fitted PL risetime values (shown in Supporting Information, Table S1) indicate the nearly linear dependence of the risetime constants with the separation distance along the microwire as will be appropriate for a step-by-step, radiative energy transfer process. Taken together, spectral and dynamics data point to photon recycling, assisted by waveguiding in $\mathrm{CsPbr}_{3}$ perovskite microwires. 
For such a scenario to occur, microwire samples must have sufficiently high PL emission quantum yield that would allow for a number of sequential absorption/re-emission events. However, the PLQY of an individual microwire cannot be reliably deduced from the bulk QY measurements. In a bulk sample, the emitted PL photons are waveguided within the microwires and experience multiple absorption/emission/scattering steps, greatly reducing their probability to escape towards detection. Standard measurements with integrating sphere provide PLQY of the microwires on the order of 5-8\%, in agreement with bulk $\mathrm{CsPbBr}_{3}$ single crystals, but clearly too small to explain the observed experimental data within the scope of photon recycling model. Furthermore, it is well known that PLQY in perovskite materials is fluence dependent. ${ }^{53}$ Measurements using integrating sphere operate at low fluence conditions where monomolecular (trap-assisted) recombination regime results in lower PLQY values. On the contrary, photon recycling necessarily involves higher excitation fluences to generate enough photons to be recorded at the remote locations along the wire. Light propagation inside the microwire is governed by the TIR light confinement factor, $T$. Following the calculations presented by Shurcliff ${ }^{54}$ for light from an isotropic emitter trapped within the rectangular waveguide of the index $n_{p e r} \sim 2.3$ with five sides facing air $\left(n_{\text {air }}=1\right)$ and one side the quartz substrate $\left(n_{q}=1.5\right)$, the confinement factor is
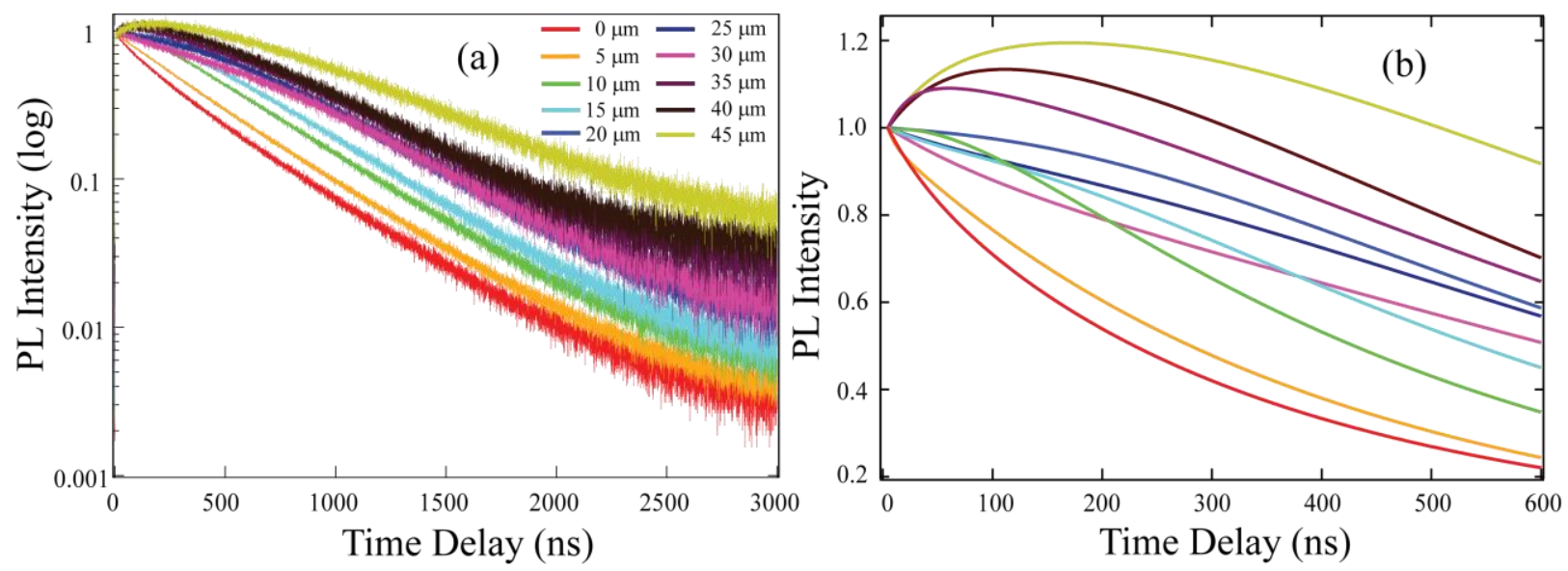

Figure 3: (a) Normalized PL dynamics of the $\mathrm{CsPbBr}_{3}$ perovskite microwires as function of separation between excitation and collection spots measured at main emission peak of $530 \mathrm{~nm}$. (b) Expanded view of the early times, presented on the linear scale, fitted curves are shown. 
estimated as $T \sim 0.63$. (i.e. once generated, $63 \%$ of the photons will forever propagate inside via TIR if no re-absorption is taking place.) Hence, in the process of photon recycling that involves multiple absorption/re-emission steps, confinement plays a major role in retaining photons within the waveguide and maintaining high enough re-excitation fluence.

In order to rationalize the observed experimental behavior of separation-dependent PL kinetics as observed in Figure 3, we analyze here an effective one-dimensional model, appropriately modified from the one found in kinetic theory of semiconductors: ${ }^{55}$

$$
\frac{\partial n(x, t)}{\partial t}=D \frac{\partial^{2} n(x, t)}{\partial x^{2}}-\frac{n(x, t)}{\tau_{\operatorname{tr}}}-B n^{2}(x, t)+q B \int d x^{\prime} K\left(\left|x-x^{\prime}\right|\right) n^{2}\left(x^{\prime}, t\right) .
$$

The validity of the one-dimensional model here is based on the overall geometrical shape of the excited region of the microwires. The initial excitation takes place via a two-photon absorption whose linear absorption length is much-much larger than the lateral dimension of the wire. The initial excitation region is therefore practically uniformly distributed across the micro wire. This uniformity is then of course preserved in the process of the diffusion making the latter a function of only coordinate $x$ along the microwire (in full similarity with textbook treatments of the diffusion from uniformly excited surface). Equation (1) thus describes the spatial-temporal evolution $n(x, t)$ of the charge carriers' density along the microwire from the initial profile $n(x, 0)$ for the equal populations of photoexcited electrons, $n$, and holes, $p$. In addition to single-particle processes of carrier diffusion (coefficient $D$ ) and trap-assisted decay (lifetime $\tau_{\text {tr }}$ ), Eq. (1) incorporates bimolecular (electronhole, $n p=n^{2}$ ) radiative recombination (coefficient $B$ ) responsible for PL emission. ${ }^{54,56}$ The last term in Eq. (1) is representative of photon recycling and describes carrier generation at point $x$ due to reabsorption of the light emitted as a result of radiative bimolecular recombination at point $x^{\prime}$ (kernel $\left.K\left(\left|x-x^{\prime}\right|\right)\right)$. As the two PL-related terms are both proportionate to $n^{2}(x, t)$, their role in the overall dynamics increases for higher excitation densities. Since bimolecular recombination 
can also involve a non-radiative channel, dimensionless parameter $0<q<1$ in Eq. (1) defines its radiative quantum yield: at $q=1$, all electron-hole recombination events result in photon emission.

To experimentally validate the bimolecular dependence of PL $\sim n^{2}$ on density $n$, we recorded initial PL intensity as a function of the laser excitation power $P$. Due to the two-photon excitation method used in this work, the light absorption itself is also proportional to the square of the laser

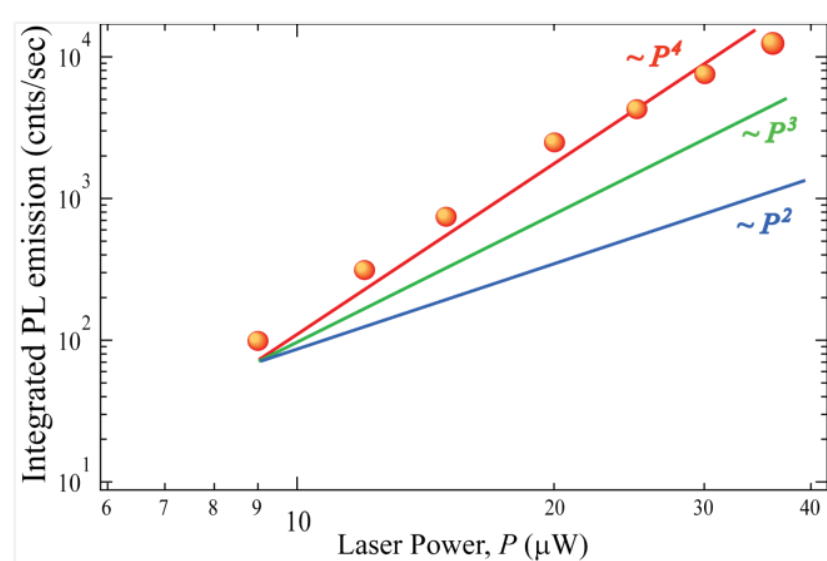

Figure 4. Initial PL intensity integrated along the microwire as a function of the applied laser power. Dots - experimental points, colored lines - proportionality to different powers of $P$. power, leading to the fourth-power dependence of the observed PL, $\sim P^{4}$ for the bimolecular recombination emission. Figure 4 shows the total PL emission, integrated along the microwire as a function of the applied $800 \mathrm{~nm}$ laser power. The calculation method as well as representative PL kinetics are shown in Supporting Information Figure S5. The observed PL signal dependence clearly follows $\sim P^{4}$ behavior, confirming the $n^{2}$ dependence of the rate of radiative processes on the density $n$ (which is different from the linear dependence, expected in the case of exciton density ${ }^{57}$ ).

The simulations resulting from the numerical solutions to the model of Eq. (1) are exemplified in Figure 5 for a set of representative parameter values. If all emitted photons were reflected from the microwire sidewalls, the spatial kernel would behave as $K(x)=\alpha \int_{1}^{\infty} e^{-\alpha|x| u} d u / 2 u$ and 


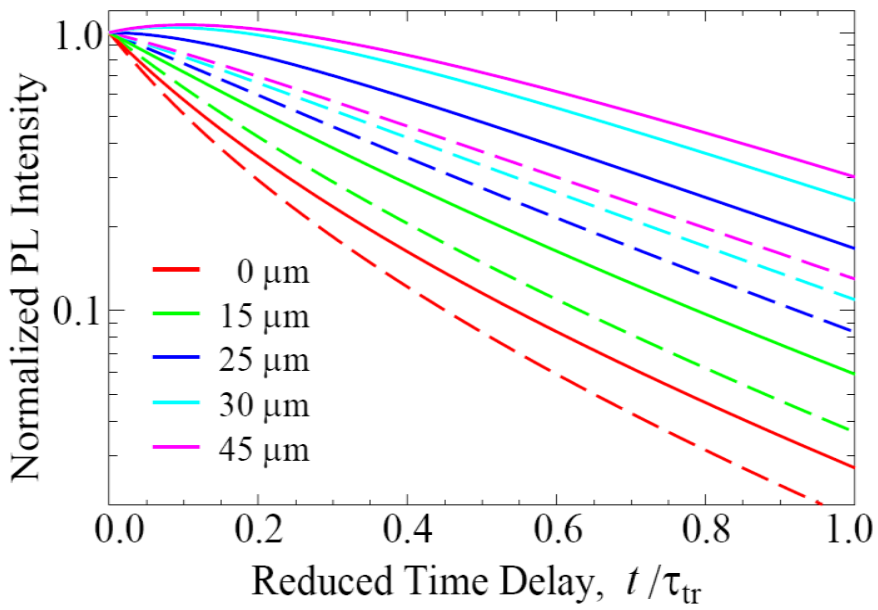

Figure 5: Model PL decay curves for several separation distances calculated with Eq. (1) and distinguished by curve colors. Compared are results with bimolecular radiative efficiency $q=0.25$ (dashed lines) and $q=1$ (solid lines). For this computational example, the maximum initial carrier density $n_{\max }=3 / B \tau_{\text {tr }}$ was used, and other parameters are defined in the text. Reduced time is shown in the units of the charge carrier lifetime.

satisfy the conservation condition, $\int_{-\infty}^{\infty} K(x) d x=1 .{ }^{58}$ We correspondingly modify the kernel to account for photons escaping through the sidewalls (and actually contributing to the observed PL); in the computational example of Figure 5, the kernel satisfies $\int_{-\infty}^{\infty} K(x) d x \cong 0.69$. This number is very close to the previously estimated TIR light confinement factor $T \sim 0.63$, while the linear absorption length $1 / \alpha=40 \mu \mathrm{m}$. (Note that this length is also much larger than the lateral size of the wire.) The model PL decay curves in Figure 5 were simulated starting from the initial excitation profile extracted from the experimental data for Figure 3. In our simulations, we purposefully used a substantially increased (with respect to the experimentally inferred) value of the single-carrier diffusion length $L_{D}=\sqrt{D \tau_{\mathrm{tr}}}=1 \mu \mathrm{m}$, and observed that, even with this value, such diffusion cannot account for the experimentally observed time-dependent spreading of the initial profile. Moreover, the observed pattern of position-dependent PL decay could not be simulated with low to moderate values of the bimolecular radiative efficiency parameter $q$ (dashed lines in Figure 5 show simulation results for $q=0.25$ ). Only for high radiative efficiency, simulation results in Figure 5 (only $q=1$ data shown by solid lines) compare favorably with experimental data in Figure 3, reflecting both faster PL decays at the early times at shorter spatial separations (associated with $n^{2}(x, t)$ term) and appearance of the delayed PL risetimes due to photon recycling at longer separations. 
Prior studies performed with methylammonium-based (MAP) perovskites have indicated that the efficiencies of bi-molecular recombination span wide range of values, from $<10 \%$ percent to close to unity and could be strongly influenced by trap-assisted Auger recombination. ${ }^{8-11}$ Consequently, this mechanism would be more relevant to polycrystalline MAP films than for single crystal materials as the latter generally have lower defect densities. Hence, our findings that bi-molecular recombination efficiency in $\mathrm{CsPbBr}_{3}$ crystalline microwires is close to unity in elementary electron-hole recombination events is in line with those models.

Our model calculations also account for competition with single-particle recombination (lifetime $\tau_{\text {tr }}$ ), their interplay being dependent on the carrier density. In our experiments, as the initial excitation profile spreads in space and decays in time, this density keeps decreasing so that eventually the single-particle recombination becomes the dominant mechanism wherever the density is low enough. Concomitantly, transient analysis reflects distance-dependent changes in the PL emission kinetics, influenced by initial carrier density and photon recycling. The high value of the radiative efficiency of the intrinsic elementary electron-hole recombination steps that is inferred from our data on the $\mathrm{CsPBr}_{3}$ microwires should thus not be confused with standard bulk measurements of the quantum yield that could result in significantly reduced - and intensity dependent - values both due to single-particle trapping and multiple reabsorption/emission steps..

Conclusions: We investigated $\mathrm{PL}$ evolution and emission dynamics in individual $\mathrm{CsPbBr}_{3}$ microwires using two-photon excitation and double objective excitation/collection microscopy. Our results show propagation of the PL emission within individual microwires to distances in excess of a $100 \mu \mathrm{m}$ from the excitation location. We found that PL spectral content and PL kinetics strongly depend on the propagation distance along the microwire. As the separation distance 
increases, the PL emission spectrally redistributes towards the lower energy while PL kinetics develop delayed emission rises, with risetime components scaling with the separation distance. We applied the one-dimensional model of spatio-temporal evolution of the charge carrier density that accounts for monomolecular and bimolecular recombination and waveguide-assisted photon self-absorption/re-emission. Simulated PL dynamics clearly show that the low PLQY values found by the bulk sample measurements are incompatible with the experimental data whenever the material experiences strong photon recycling. The model reveals internal recombination efficiency of the microwires to be close to unity. These findings show the influence of photon recycling on the emission properties of $\mathrm{CsPBrr}_{3}$ perovskites and underscore its significance for rational design of cesium halide perovskite materials for light emitting and photon conversion applications.

Supporting Information. This material is available free of charge via the Internet at http://pubs.acs.org. Experimental sections, EDX, optical images of microwires, fitted PL kinetics and mobility measurements (SCLC and FET).

\section{AUTHOR INFORMATION}

\section{Corresponding Authors:}

*anton.malko@utdallas.edu

*omar.abdelsaboor@kaust.edu.sa

\section{Author Contributions}

The manuscript was written through contributions of all authors. All authors have given approval to the final version of the manuscript.

\section{ACKNOWLEDGMENT}


(1) Tsai, H.; Nie, W.; Blancon, J.-C.; Stoumpos, C. C.; Asadpour, R.; Harutyunyan, B.; Neukirch, A. J.; Verduzco, R.; Crochet, J. J.; Tretiak, S.; et al. High-Efficiency Two-Dimensional Ruddlesden-Popper Perovskite Solar Cells. Nature 2016, 536, 312-316.

(2) Cho, H.; Jeong, S.-H.; Park, M.-H.; Kim, Y.-H.; Wolf, C.; Lee, C.-L.; Heo, J. H.; Sadhanala, A.; Myoung, N.; Yoo, S.; et al. Overcoming the Electroluminescence Efficiency Limitations of Perovskite Light-Emitting Diodes. Science 2015, 350, 1222-1225.

(3) Pathak, S.; Sakai, N.; Wisnivesky Rocca Rivarola, F.; Stranks, S. D.; Liu, J.; Eperon, G. E.; Ducati, C.; Wojciechowski, K.; Griffiths, J. T.; Haghighirad, A. A.; et al. Perovskite Crystals for Tunable White Light Emission. Chem. Mater. 2015, 27, 8066-8075.

(4) De Bastiani, M.; Saidaminov, M. I.; Dursun, I.; Sinatra, L.; Peng, W.; Buttner, U.; Mohammed, O. F.; Bakr, O. M. Thermochromic Perovskite Inks for Reversible Smart Window Applications. Chem. Mater. 2017, 29, 3367-3370.

(5) National Renewable Energy Laboratory. Best Research-Cell Efficiencies. http://www.nrel.gov/ncpv/images/efficiency_chart.jpg (Accesed:April, 2018).

(6) Zhu, H.; Fu, Y.; Meng, F.; Wu, X.; Gong, Z.; Ding, Q.; Gustafsson, M. V.; Trinh, M. T.; Jin, S.; Zhu, X. Y. Lead Halide Perovskite Nanowire Lasers with Low Lasing Thresholds and High Quality Factors. Nat. Mater. 2015, 14, 636-642.

(7) Zhou, H.; Chen, Q.; Li, G.; Luo, S.; Song, T.-b.; Duan, H.-S.; Hong, Z.; You, J.; Liu, Y.; Yang, Y. Interface Engineering of Highly Efficient Perovskite Solar Cells. Science 2014, 345, 542-546.

(8) Richter, J. M.; Abdi-Jalebi, M.; Sadhanala, A.; Tabachnyk, M.; Rivett, J. P. H.; Pazos-Outón, L. M.; Gödel, K. C.; Price, M.; Deschler, F.; Friend, R. H., Enhancing Photoluminescence Yields in Lead Halide Perovskites by Photon Recycling and Light Out-coupling. Nat. Commun. 2016, 7 , 13941.

(9) Crothers, T. W.; Milot, R. L.; Patel, J. B.; Parrott, E. S.; Schlipf, J.; Müller-Buschbaum, P.; Johnston, M. B.; Herz, L. M., Photon Reabsorption Masks Intrinsic Bimolecular Charge-Carrier Recombination in $\mathrm{CH}_{3} \mathrm{NH}_{3} \mathrm{PbI}_{3}$ Perovskite. Nano Lett. 2017, 17, 5782-5789.

(10) Staub, F.; Kirchartz, T.; Bittkau, K.; Rau, U., Manipulating the Net Radiative Recombination Rate in Lead Halide Perovskite Films by Modification of Light Outcoupling. J. Phys. Chem. Lett. 2017, 8, 5084-5090. 
(11) Ha, S.-T.; Shen, C.; Zhang, J.; Xiong, Q., Laser Cooling of Organic-Inorganic Lead Halide Perovskites. Nat. Photonics 2015, 10, 115-121.

(12) Stranks, S. D.; Snaith, H. J. Metal-Halide Perovskites for Photovoltaic and Light-Emitting Devices. Nat Nanotechnol. 2015, 10, 391-402.

(13) Stranks, S. D.; Eperon, G. E.; Grancini, G.; Menelaou, C.; Alcocer, M. J. P.; Leijtens, T.; Herz, L. M.; Petrozza, A.; Snaith, H. J. Electron-Hole Diffusion Lengths Exceeding 1 Micrometer in an Organometal Trihalide Perovskite Absorber. Science 2013, 342, 341-344.

(14) Xing, G.; Mathews, N.; Sun, S.; Lim, S. S.; Lam, Y. M.; Grätzel, M.; Mhaisalkar, S.; Sum, T. C. Long-Range Balanced Electron- and Hole-Transport Lengths in Organic-Inorganic $\mathrm{CH}_{3} \mathrm{NH}_{3} \mathrm{PbI}_{3}$. Science 2013, 342, 344-347.

(15) Yang, H.; Zhang, Y.; Pan, J.; Yin, J.; Bakr, O. M.; Mohammed, O. F., Room-Temperature Engineering of All-Inorganic Perovskite Nanocrsytals with Different Dimensionalities. Chem. Mater. 2017, 29, 8978-8982.

(16) Yin, J.; Zhang, Y.; Bruno, A.; Soci, C.; Bakr, O. M.; Brédas, J.-L.; Mohammed, O. F., Intrinsic Lead Ion Emissions in Zero-Dimensional $\mathrm{Cs}_{4} \mathrm{PbBr}_{6}$ Nanocrystals. ACS Energy Lett. 2017, 2, 2805 2811.

(17) Zhang, Y.; Saidaminov, M. I.; Dursun, I.; Yang, H.; Murali, B.; Alarousu, E.; Yengel, E.; Alshankiti, B. A.; Bakr, O. M.; Mohammed, O. F., Zero-Dimensional $\mathrm{Cs}_{4} \mathrm{PbBr}_{6} \mathrm{Perovskite}$ Nanocrystals. J. Phys. Chem. Lett. 2017, 8, 961-965.

(18) Yin, J.; Maity, P.; De Bastiani, M.; Dursun, I.; Bakr, O. M.; Brédas, J.-L.; Mohammed, O. F., Molecular Behavior of Zero-Dimensional Perovskites. Sci. Adv. 2017, 3, e1701793.

(19) Ahmed, G. H.; Yin, J.; Bose, R.; Sinatra, L.; Alarousu, E.; Yengel, E.; AlYami, N. M.; Saidaminov, M. I.; Zhang, Y.; Hedhili, M. N.; et al. Pyridine-Induced Dimensionality Change in Hybrid Perovskite Nanocrystals. Chem. Mater. 2017, 29, 4393-4400.

(20) De Bastiani, M.; Dursun, I.; Zhang, Y.; Alshankiti, B. A.; Miao, X.-H.; Yin, J.; Yengel, E.; Alarousu, E.; Turedi, B.; Almutlaq, J. M.; et al. Inside Perovskites: Quantum Luminescence from Bulk $\mathrm{Cs}_{4} \mathrm{PbBr}_{6}$ Single Crystals. Chem. Mater. 2017, 29, 7108-7113.

(21) Dursun, I.; De Bastiani, M.; Turedi, B.; Alamer, B.; Shkurenko, A.; Yin, J.; El-Zohry, A. M.; Gereige, I.; AlSaggaf, A.; Mohammed, O. F.; Eddaoudi, M.; Bakr, O. M., CsPb ${ }_{2} \mathrm{Br}_{5} \mathrm{Single}$ Crystals: Synthesis and Characterization. ChemSusChem 2017, 10, 3746-3749.

(22) Xing, G.; Mathews, N.; Lim, S. S.; Yantara, N.; Liu, X.; Sabba, D.; Grätzel, M.; Mhaisalkar, S.; Sum, T. C. Low-Temperature Solution-Processed Wavelength-Tunable Perovskites for Lasing. Nat. Mater. 2014, 13, 476-480.

(23) Veldhuis, S. A.; Boix, P. P.; Yantara, N.; Li, M.; Sum, T. C.; Mathews, N.; Mhaisalkar, S. G. Perovskite Materials for Light-Emitting Diodes and Lasers. Adv. Mater. 2016, 28, 6804-6834.

(24) Yantara, N.; Bhaumik, S.; Yan, F.; Sabba, D.; Dewi, H. A.; Mathews, N.; Boix, P. P.; Demir, H. V.; Mhaisalkar, S. Inorganic Halide Perovskites for Efficient Light-Emitting Diodes. J. Phys. Chem. Lett. 2015, 6, 4360-4364.

(25) Yakunin, S.; Protesescu, L.; Krieg, F.; Bodnarchuk, M. I.; Nedelcu, G.; Humer, M.; De Luca, G.; Fiebig, M.; Heiss, W.; Kovalenko, M. V. Low-Threshold Amplified Spontaneous Emission and Lasing from Colloidal Nanocrystals of Caesium Lead Halide Perovskites. Nat. Commun. 2015, $6,8056$.

(26) Fu, Y.; Zhu, H.; Stoumpos, C. C.; Ding, Q.; Wang, J.; Kanatzidis, M. G.; Zhu, X.; Jin, S. Broad Wavelength Tunable Robust Lasing from Single-Crystal Nanowires of Cesium Lead Halide Perovskites $\left(\mathrm{CsPbX}_{3}, \mathrm{X}=\mathrm{Cl}, \mathrm{Br}, \mathrm{I}\right)$. ACS Nano 2016, 10, 7963-7972. 
(27) Eaton, S. W.; Lai, M.; Gibson, N. A.; Wong, A. B.; Dou, L.; Ma, J.; Wang, L.-W.; Leone, S. R.; Yang, P. Lasing in Robust Cesium Lead Halide Perovskite Nanowires. Proc. Natl. Acad. Sci. U.S.A. 2016, 113, 1993-1998.

(28) Dirin, D. N.; Cherniukh, I.; Yakunin, S.; Shynkarenko, Y.; Kovalenko, M. V. Solution-Grown $\mathrm{CsPbBr}_{3}$ Perovskite Single Crystals for Photon Detection. Chem. Mater. 2016, 28, 8470-8474.

(29) Saidaminov, M. I.; Haque, M. A.; Almutlaq, J.; Sarmah, S.; Miao, X.-H.; Begum, R.; Zhumekenov, A. A.; Dursun, I.; Cho, N.; Murali, B.; et al. Inorganic Lead Halide Perovskite Single Crystals: Phase-Selective Low-Temperature Growth, Carrier Transport Properties, and SelfPowered Photodetection. Adv. Opt. Mat. 2017, 5, 1600704.

(30) Imran, M.; Di Stasio, F.; Dang, Z.; Canale, C.; Khan, A. H.; Shamsi, J.; Brescia, R.; Prato, M.; Manna, L. Colloidal Synthesis of Strongly Fluorescent $\mathrm{CsPbBr}_{3}$ Nanowires with Width Tunable down to the Quantum Confinement Regime. Chem. Mater. 2016, 28, 6450-6454. (31) Zhang, D.; Eaton, S. W.; Yu, Y.; Dou, L.; Yang, P. Solution-Phase Synthesis of Cesium Lead Halide Perovskite Nanowires. J. Am. Chem. Soc. 2015, 137, 9230-9233.

(32) Wang, Y.; Sun, X.; Shivanna, R.; Yang, Y.; Chen, Z.; Guo, Y.; Wang, G.-C.; Wertz, E.; Deschler, F.; Cai, Z.; et al. Photon Transport in One-Dimensional Incommensurately Epitaxial $\mathrm{CsPbX}_{3}$ Arrays. Nano Lett. 2016, 16, 7974-7981.

(33) Protesescu, L.; Yakunin, S.; Bodnarchuk, M. I.; Krieg, F.; Caputo, R.; Hendon, C. H.; Yang, R. X.; Walsh, A.; Kovalenko, M. V. Nanocrystals of Cesium Lead Halide Perovskites $\left(\mathrm{CsPb}_{3}, \mathrm{X}\right.$ $=\mathrm{Cl}, \mathrm{Br}$, and I): Novel Optoelectronic Materials Showing Bright Emission with Wide Color Gamut. Nano Lett. 2015, 15, 3692-3696.

(34) Zhang, Y.; Sinatra, L.; Alarousu, E.; Yin, J.; El-Zohry, A. M.; Bakr, O. M.; Mohammed, O. F. Ligand-Free Nanocrystals of Highly Emissive $\mathrm{Cs}_{4} \mathrm{PbBr}_{6}$ Perovskite. J. Phys. Chem. C 2018, 122, 6493-6498.

(35) Wang, S.; Wang, K.; Gu, Z.; Wang, Y.; Huang, C.; Yi, N.; Xiao, S.; Song, Q. Solution-Phase Synthesis of Cesium Lead Halide Perovskite Microrods for High-Quality Microlasers and Photodetectors. Adv. Opt. Mater. 2017, 5, 1700023.

(36) Asbeck, P. Self-Absorption Effects on the Radiative Lifetime in GaAs-GaAlAs Double Heterostructures. J. of Appl. Phys. 1977, 48, 820-822.

(37) Ahrenkiel, R. K.; Dunlavy, D. J.; Keyes, B.; Vernon, S. M.; Dixon, T. M.; Tobin, S. P.; Miller, K. L.; Hayes, R. E. Ultralong Minority-Carrier Lifetime Epitaxial GaAs by Photon Recycling. App. Phys. Lett. 1989, 55, 1088-1090.

(38) Renaud, P.; Raymond, F.; Bensaïd, B.; Vèrié, C. Influence of Photon Recycling on Lifetime and Diffusion Coefficient in GaAs. J. Appl. Phys. 1992, 71, 1907-1913.

(39) Stoita, A.; Vautey, T.; Jacquier, B.; Guy, S. Impact of the Radiation Trapping on Lifetime Measurement of $\mathrm{Er}^{3+}$ Doped glasses. J. Lumin. 2010, 130, 1119-1123.

(40) Caird, J. A.; Ramponi, A. J.; Staver, P. R. Quantum Efficiency and Excited-State Relaxation Dynamics in Neodymium-Doped Phosphate Laser Glasses. J. Opt. Soc. Am. B 1991, 8, 1391-1403. (41) Miller, O. D.; Yablonovitch, E.; Kurtz, S. R. Strong Internal and External Luminescence as Solar Cells Approach the Shockley-Queisser Limit. IEEE J. Photovoltaics 2012, 2, 303-311.

(42) Dupont, E.; Liu, H. C.; Buchanan, M.; Chiu, S.; Gao, M. Efficient GaAs Light-Emitting Diodes by Photon Recycling. Appl. Phys. Lett. 2000, 76, 4-6.

(43) Pazos-Outón, L. M.; Szumilo, M.; Lamboll, R.; Richter, J. M.; Crespo-Quesada, M.; AbdiJalebi, M.; Beeson, H. J.; Vrućinić, M.; Alsari, M.; Snaith, H. J.; et al. Photon Recycling in Lead Iodide Perovskite Solar Cells. Science 2016, 351, 1430-1433. 
(44) Yablonovitch, E. Lead Halides Join the Top Optoelectronic League. Science 2016, 351, 14011401.

(45) Fang, Y.; Wei, H.; Dong, Q.; Huang, J. Quantification of Re-Absorption and Re-Emission Processes to Determine Photon Recycling Efficiency in Perovskite Single Crystals. Nat. Commun. 2017, 8, 14417.

(46) Grancini, G.; D'Innocenzo, V.; Dohner, E. R.; Martino, N.; Srimath Kandada, A. R.; Mosconi, E.; De Angelis, F.; Karunadasa, H. I.; Hoke, E. T.; Petrozza, A. $\mathrm{CH}_{3} \mathrm{NH}_{3} \mathrm{PbI}_{3}$ Perovskite Single Crystals: Surface Photophysics and Their Interaction with the Environment. Chem. Sci. 2015, 6, 7305-7310.

(47) Alarousu, E.; El-Zohry, A. M.; Yin, J.; Zhumekenov, A. A.; Yang, C.; Alhabshi, E.; Gereige, I.; AlSaggaf, A.; Malko, A. V.; Bakr, O. M.; et al. Ultralong Radiative States in Hybrid Perovskite Crystals: Compositions for Submillimeter Diffusion Lengths. J. Phys. Chem. Lett. 2017, 8, 43864390.

(48) Nguyen, V.-C.; Katsuki, H.; Sasaki, F.; Yanagi, H. Optically Pumped Lasing in Single Crystals of Organometal Halide Perovskites Prepared by Cast-Capping Method. Appl. Phys. Lett. 2016, 108, 261105.

(49) Guy, S. Modelization of Lifetime Measurement in the Presence of Radiation Trapping in Solid-State Materials. Phys. Rev. B 2006, 73, 144101.

(50) Sarmah, S. P.; Burlakov, V. M.; Yengel, E.; Murali, B.; Alarousu, E.; El-Zohry, A. M.; Yang, C.; Alias, M. S.; Zhumekenov, A. A.; Saidaminov, M. I.; et al. Double Charged Surface Layers in Lead Halide Perovskite Crystals. Nano Lett. 2017, 17, 2021-2027.

(51) Tian, W.; Zhao, C.; Leng, J.; Cui, R.; Jin, S. Visualizing Carrier Diffusion in Individual Single-Crystal Organolead Halide Perovskite Nanowires and Nanoplates. J. Am. Chem. Soc. 2015, $137,12458-12461$.

(52) Huo, C.; Liu, X.; Song, X.; Wang, Z.; Zeng, H., Field-Effect Transistors Based on van-derWaals-Grown and Dry-Transferred All-Inorganic Perovskite Ultrathin Platelets. J. Phys. Chem. Lett. 2017, 8, 4785-4792.

(53) Manser, J. S.; Kamat, P. V. Band Filling with Free Charge Carriers in Organometal Halide Perovskites. Nat. Photonics 2014, 8, 737-743.

(54) Shurcliff, W. A.; Jones, R. C. The Trapping of Fluorescent Light Produced within Objects of High Geometrical Symmetry. J. Opt. Soc. Am. 1949, 39, 912-916.

(55) Sze, S. M.; Ng, K. K. Physics of Semiconductor Devices, $3^{\text {rd }}$ ed; Wiley-Interscience: Hoboken, NJ, 2006; pp 5-75.

(56) Stranks, S. D.; Burlakov, V. M.; Leijtens, T.; Ball, J. M.; Goriely, A.; Snaith, H. J. Recombination Kinetics in Organic-Inorganic Perovskites: Excitons, Free Charge, and Subgap States. Phys. Rev. Appl. 2014, 2, 034007.

(57) Agranovich, V. M. and Galanin, M. D. Electronic Excitation Energy Transfer in Condensed Matter. Nauka:Moscow, 1978. 